\title{
Gamete Formation Resets the Aging Clock in Yeast
}

\author{
E. ÜNAL AND A. Amon \\ David H. Koch Institute for Integrative Cancer Research, Howard Hughes Medical Institute, \\ Massachusetts Institute of Technology, Cambridge, Massachusetts 02142 \\ Correspondence: angelika@mit.edu
}

\begin{abstract}
Gametogenesis is a process whereby a germ cell differentiates into haploid gametes. We found that, in budding yeast, replicatively aged cells remove age-induced cellular damage during gametogenesis. Importantly, gametes of aged cells have the same replicative potential as those derived from young cells, indicating that life span resets during gametogenesis. Here, we explore the potential mechanisms responsible for gametogenesis-induced rejuvenation and discuss putative analogous mechanisms in higher eukaryotes.
\end{abstract}

Most organisms, if not all, are susceptible to aging, a process characterized by the deterioration of subcellular structures, increased genomic instability, decreased protein homeostasis, and accumulation of reactive oxygen species (Johnson et al. 1999). Despite the dramatic impact of aging on an organism, age-associated traits are not passed on to the progeny. One model put forth to explain how the offspring is protected from the detrimental effects of aging is based on the immortal germline-disposable soma theory (Kirkwood 1987). In this model the germline, the cell lineage from which reproductive cells are derived, is distinct from the somatic cells in that they are protected from aging. This hypothesis implies that a set of cells or nuclei is predetermined to give rise to the germline very early during embryogenesis and protected from aging.

Studies in Drosophila melanogaster argue against this hypothesis. In Drosophila, the first 13 nuclear divisions of embryogenesis occur within a common cytoplasm, called the syncytium, with maternally deposited RNAs directing development of the organism. At the posterior pole, maternally deposited polar granules, RNA-protein particles, instruct nuclei in their vicinity to become germ cells. When these polar granules are transplanted or directed to be deposited at the anterior end of the embryo, germ cells will develop at the anterior end of the embryo (Ephrussi and Lehmann 1992). Thus, any nucleus within the syncytium, provided that it is in the vicinity of polar granules, can become a germ cell. These findings argue against preexisting cell-autonomous factors in the germline that provide immunity against aging.

If germ cells are not protected from aging, mechanisms must exist that reset life span from one generation to the next. Resetting could occur during gametogenesis or embryogenesis. Using the budding yeast, Saccharomyces cerevisiae, we tested the hypothesis that resetting of life span occurs during gametogenesis (Unal et al. 2011). Here we will summarize our recent findings that suggest that in budding yeast gametogenesis erases age-associated phenotypes and resets life span. We will then discuss potential approaches to identify the mechanism(s) responsible for gametogenesis-induced rejuvenation. Finally, we will discuss the possibility that similar resetting mechanisms also occur in multicellular organisms.

\section{GAMETOGENESIS CAUSES REJUVENATION IN BUDDING YEAST}

To test the hypothesis that resetting of life span occurs during gametogenesis, we chose budding yeast as our experimental system for the following reasons: First, gamete formation, sporulation in yeast, can be readily induced by nutrient deprivation (Marston and Amon 2004). Second, budding yeast cells undergo replicative aging; each cell produces a finite number of progeny (Mortimer and Johnston 1959). Finally, both young and aged cells can be isolated easily (Sinclair et al. 1998) and followed throughout sporulation.

The replicative life span of a yeast cell is measured by the total number of cell divisions a single (mother) cell undergoes before its death (Sinclair et al. 1998). In a given strain background, the average replicative life span of a mother cell population is relatively constant, allowing one to make reliable measurements of conditions or genetic alterations that extend or shorten life span (Sinclair et al. 1998). Yeast cells also experience a different life span known as the chronological life span. This life span describes the length of time a yeast cell can survive in G0 (Fabrizio and Longo 2003). Here, we will solely discuss replicative life span and refer to this simply as "life span."

In budding yeast, gametes are referred to as spores. Sporulation necessitates a specialized cell division called meiosis and a morphogenetic program for spore formation (Marston and Amon 2004). Meiosis causes a reduction of the diploid to the haploid state. The resulting 
nuclei are then packaged into spores. Spore formation includes the generation of new membrane compartments within the cell's cytoplasm, extensive protein and organelle degradation, and synthesis of a spore wall that provides resistance to environmental stresses (Neiman 2005).

To determine whether gamete formation causes rejuvenation, we asked whether spores that are derived from aged cells have reset their life span and are young, or whether they inherit the progenitor's age and remain old. This analysis revealed that, upon sporulation, the life spans of the spores derived from young and aged cells were indistinguishable from each other, whereas the aged cells not induced to form spores died rapidly (Unal et al. 2011). Importantly, all four spores from a given tetrad entered their vegetative life cycle with a full replicative potential (Unal et al. 2011). This is in contrast to mitosis, in which age is asymmetrically inherited between the mother cell and the bud, likely through processes that prevent the transmission of age-associated damage from the aging mother cell to the newly formed daughter cell (Sinclair et al. 1998). These results demonstrate that, in budding yeast, gamete formation resets replicative life span.

How does sporulation bring about resetting of life span? Yeast and metazoans share a set of aging characteristics such as increased levels of protein aggregation and carbonylation (Nystrom 2005; Morimoto 2008). In addition, aging yeast cells harbor aberrant nucleolar structures and increased levels of extra chromosomal ribosomal DNA circles (ERCs) (Sinclair and Guarente 1997; Sinclair et al. 1997). We examined these aging phenotypes in aged cells before and after sporulation.

In budding yeast, protein aggregates associate with Hsp104, a stress-tolerance chaperone, and can be seen as green fluorescent protein (GFP) foci by expressing an Hsp104-eGFP fusion in cells (Erjavec et al. 2007). In mitotic cells, these foci are distributed asymmetrically between the mother and daughter cells. This asymmetric

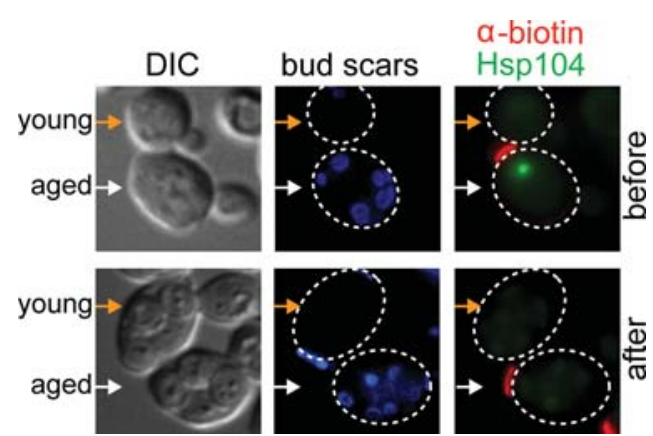

Figure 1. Hsp104-eGFP aggregates are eliminated upon sporulation. Analysis of Hsp 104-eGFP aggregates in A25825 carrying an Hsp104-eGFP fusion. To isolate aged cells, we adapted an assay based on the biotin labeling of a cell population followed by the isolation of labeled cells with streptavidin-coated magnetic beads (Smeal et al. 1996). Since the biotin molecules are retained only in the mother cells as part of the cell wall, the subsequent growth and isolation of the biotin-labeled cells allows for the enrichment of replicatively aged cells. A young (biotinnegative, highlighted by orange arrows) and an aged (biotinpositive, highlighted by white arrows) cell is shown before and after sporulation. Hsp104-eGFP aggregates are detectable as bright green foci. Biotin is labeled with streptavidin-Alexa 568 ( $\alpha$-biotin, red) and bud scars are labeled with lectin-Alexa 360 (blue). DIC, differential interference contrast. The strains used in this and the subsequent experiments are listed in Table 1.

segregation depends on the polarisome, a protein complex that directs the localized assembly of actin filaments at the polarization site (Park and Bi 2007), and Sir2, a nicotinamide adenine dinucleotide $\left(\mathrm{NAD}^{+}\right)$-dependent histone deacetylase previously implicated in replicative aging (Tessarz et al. 2009; Liu et al. 2010). Hsp104-eGFP foci were present in aged cells undergoing meiosis, but the foci were absent in mature spores (Fig. 1; Unal et al. 2011). These findings suggest that age-associated protein aggregates are cleared during late stages of sporulation, perhaps by exclusion from spores and/or degradation. We also analyzed protein carbonylation, which

Table 1. Yeast strains used in this study

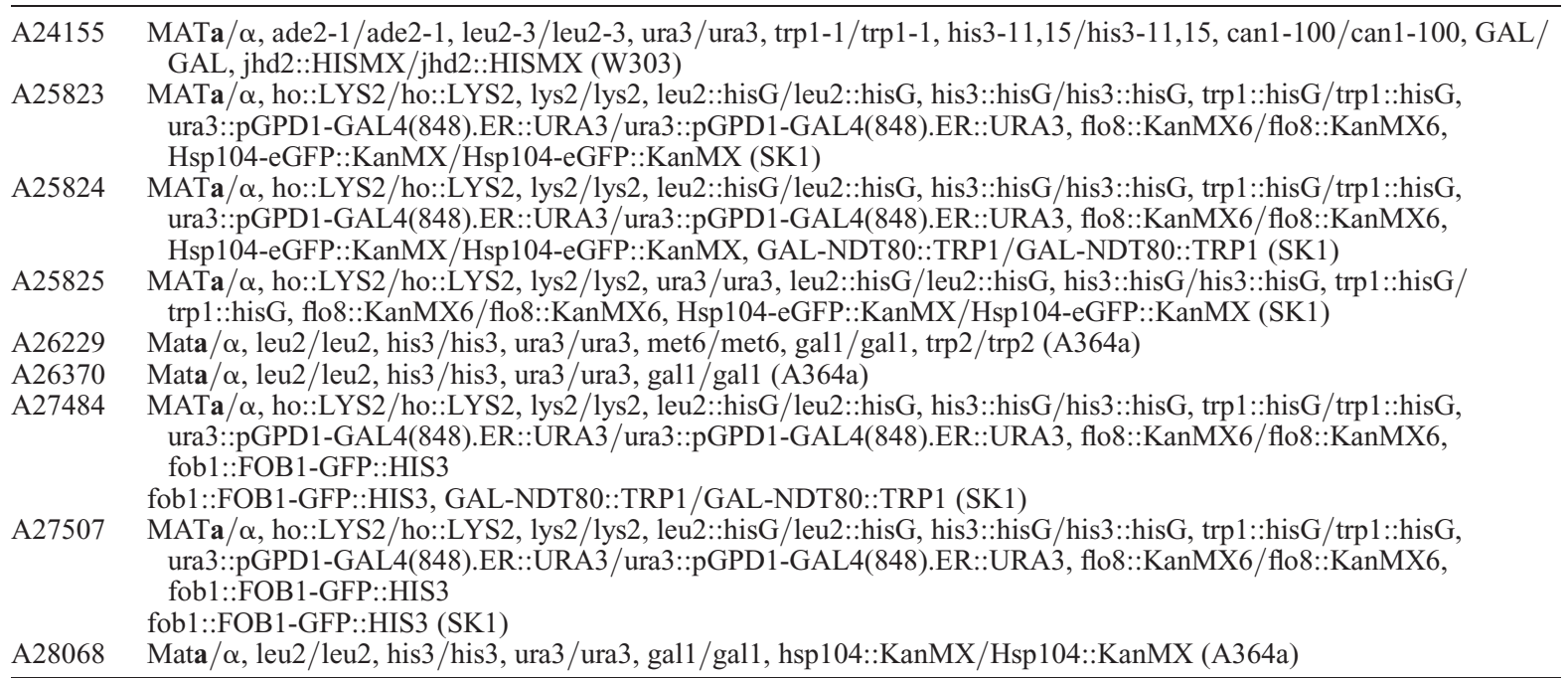




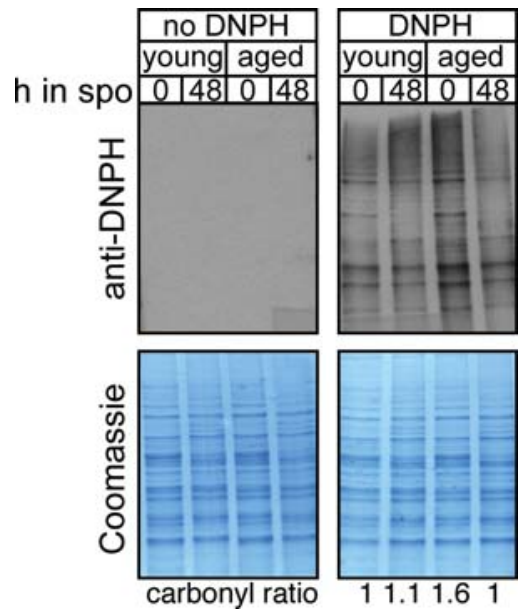

Figure 2. Protein carbonylation is decreased upon sporulation. Protein carbonylation in young $(1 \pm 0.7$ generations $)$ and aged (12 \pm 3 generations) cells from the strain A26229 at 0 or $48 \mathrm{~h}$ after sporulation. The experiments was performed as follows: $10 \mathrm{OD}_{600}$ units of frozen cells were resuspended in $50 \mathrm{mM}$ Tris $\mathrm{pH} 8,150 \mathrm{mM} \mathrm{NaCl}$, and $0.5 \% \mathrm{NP}-40$, supplemented with protease inhibitors. Cells were pulverized with glass beads using a mini bead-beater. A $15-20 \mu \mathrm{g}$ aliquot of total protein was then derivatized with dinitrophenylhydrazine (DNPH), and an identical amount of protein was incubated with a derivatizationcontrol solution (no DNPH). Carbonylated proteins were detected using the Oxyblot protein oxidation detection kit (Chemicon) following the manufacturer's instructions. To calculate the carbonylation ratios (shown below each lane), the signal from the anti-DNPH blot was first calibrated based on loading (Coomassie). This value from the $0 \mathrm{~h}$ sample of young cells was set to a ratio of 1 and the ratios for the subsequent samples were calculated accordingly.

is induced by oxidative damage and observed in age-associated diseases such as Alzheimer's disease, Parkinson's disease, and cancer (Nystrom 2005). In yeast, carbonylated proteins were reported to colocalize with Hsp104 aggregates in aged cells (Erjavec et al. 2007). We examined protein carbonylation in cell extracts by immunoblotting (Fig. 2). Prior to sporulation, we observed an approximately twofold increase in protein carbonylation in aged cells ( $12 \pm 3$ generations) compared with the young cells $(1 \pm 0.7$ generations). However, after sporulation carbonylated proteins were present at similar levels in spores obtained from aged cells as spores from young cells (Fig. 2). We conclude that damaged as well as aggregated proteins are cleared from aged cells during sporulation.

Aged cells also display nucleolar defects. Nucleoli are fragmented and illegitimate recombination results in the formation of ERCs (Sinclair and Guarente 1997; Sinclair et al. 1997). After sporulation, nucleolar morphology of spores derived from aged cells was indistinguishable from that obtained from young cells. Sporulation also reduced ERCs in aged cells (Unal et al. 2011). Taken together, these results demonstrate that gamete formation eliminates age-induced cellular damage such as protein aggregation and nucleolar aberrations.

Which aspects of sporulation bring about life span resetting? Our previous studies indicated that nutrient deprivation, a prerequisite for sporulation, is not sufficient to promote resetting of life span. Furthermore we find that pre-meiotic DNA replication and recombination are insufficient and the meiotic divisions are dispensable for rejuvenation. However, Ndt80, a transcription factor that induces the expression of genes required for the meiotic divisions and spore formation, is essential for life span resetting (Unal et al. 2011). Expression of this transcription factor during vegetative growth is also sufficient to extend the life span of cells. Interestingly, even a transient expression of NDT80 caused life span extension (Unal et al. 2011). This latter observation suggests that the gametogenesis-induced rejuvenation program can, at least in part, be induced during vegetative growth. In the next section, we discuss approaches to delineating the pathways responsible for this phenomenon.

\section{MECHANISMS OF GAMETOGENESIS- INDUCED REJUVENATION}

Our results indicate that gamete formation causes rejuvenation by eliminating age-associated traits. This rejuvenation requires the sporulation-specific transcription factor Ndt80. Expression of this transcription factor can also extend the life span of vegetatively growing cells. The latter observation is important for two reasons: First, it suggests that at least a subset of the factors required for rejuvenation during gametogenesis could function outside of this developmental program, providing a tool to identify these age-reversal mechanisms. Second, the fact that transient expression of Ndt80 can extend a cell's life span creates a unique opportunity to dissect the molecular causes of aging. We can now ask which age-associated markers are eliminated during the NDT80-mediated life span extension and which genes are required for this life span extension. Determining the fate of age-associated changes upon NDT80 induction as well as identifying and characterizing Ndt80 target genes will provide insights into this question.

To begin to address how transient induction of Ndt80 extends the life span of aged cells, we monitored the age-associated cellular changes after NDT80 induction. We first examined the possibility that Hsp104-eGFP aggregates are a cause of aging in yeast. Several lines of evidence argue against this possibility. First, Hsp104-eGFP aggregates were not reduced immediately following NDT80 induction (Unal et al. 2011). Second, Hsp104-eGFP foci still form in cells, which continuously express NDT80 from a young age onward (Fig. 3). Third, deletion of HSP104 does not significantly affect the life span of cells of the A364a strain background (Fig. 4).

In contrast to Hsp104-eGFP aggregates, nucleolar morphology is impacted by the transient expression of NDT80. The nucleolus is enlarged and fragmented in aged cells and this phenotype is reduced following the transient expression of NDT80. However, ERCs do not decline upon transient NDT80 induction, at least within the first $6 \mathrm{~h}$ after NDT80 expression (Unal et al. 2011; Fig. 5). Thus, transient induction of NDT80 causes a 


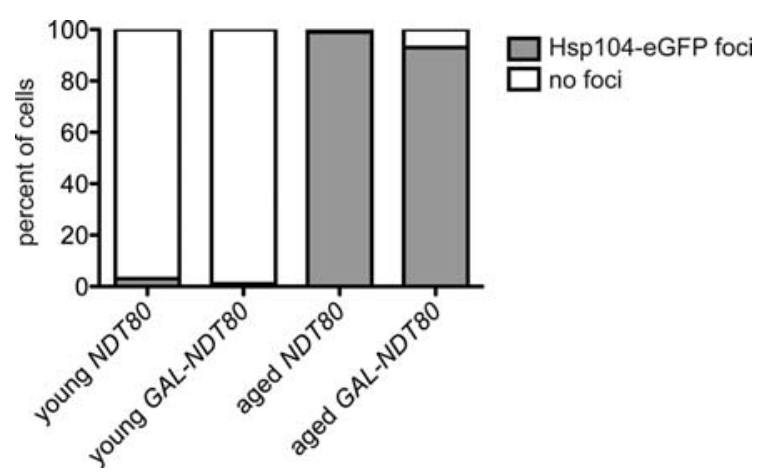

Figure 3. Hsp104-eGFP aggregates form in cells continuously expressing NDT80. Quantification of Hsp104-eGFP foci in A25823 (GAL4-ER, NDT80) or A25824 (GAL4-ER, GALNDT80) strains. In A25824, NDT80 is expressed from the GAL1-10 promoter (GAL-NDT80), whose expression can be regulated by a Gal4-estrogen receptor fusion protein (Gal4.ER). Addition of estrogen ( $\beta$-estradiol) rapidly induces expression of Ndt80 and produces Ndt80 levels similar in quantity to those observed during sporulation (Unal et al. 2011). For this experiment, the cells were aged in the presence of $\beta$-estradiol, which results in the continuous induction of GAL-NDT80. The percentage of cells with or without Hsp104-eGFP foci was determined for 100 cells. The average age for young GAL4-ER, NDT80 cells was $1.4 \pm 0.6$ generations; for aged GAL4-ER, NDT80 cells it was $12.7^{-} \pm 2.4$ generations; for young GAL4-ER, GAL-NDT80 cells it was $1.6 \pm 0.8$ generations; and for aged GAL4-ER, GAL-NDT80 cells it was $12.8 \pm 2.4$ generations.

change in nucleolar/rDNA structure, reverting it to a state that resembles that seen in young cells. Interestingly, dramatic changes in nucleolar structure are also observed during sporulation. Budding yeast cells eliminate most of the nucleolar material during spore packaging (Fuchs and Loidl 2004). These findings suggest that morphological changes in the nucleolus contribute to the NDT80-dependent life span extension and are part of sporulation-induced rejuvenation. Whether this loss of architecture is restricted to the nucleolus or occurs in the entire nucleus is not yet known.

The observation that NDT80-mediated life span extension is accompanied by recovery of nucleolar morphology, but not by removal of protein aggregates or ERCs,

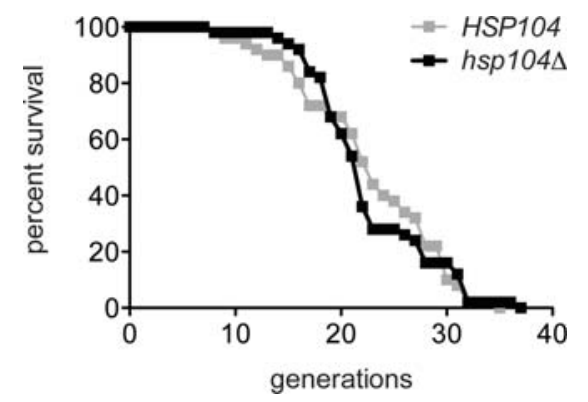

Figure 4. Deletion of HSP104 does not affect the life span of A364a cells. Pedigree of wild-type (A26370) or hsp104D (A28068) strains on YEPD plates. Fifty cells were monitored for each strain as described in Unal et al. (2011).
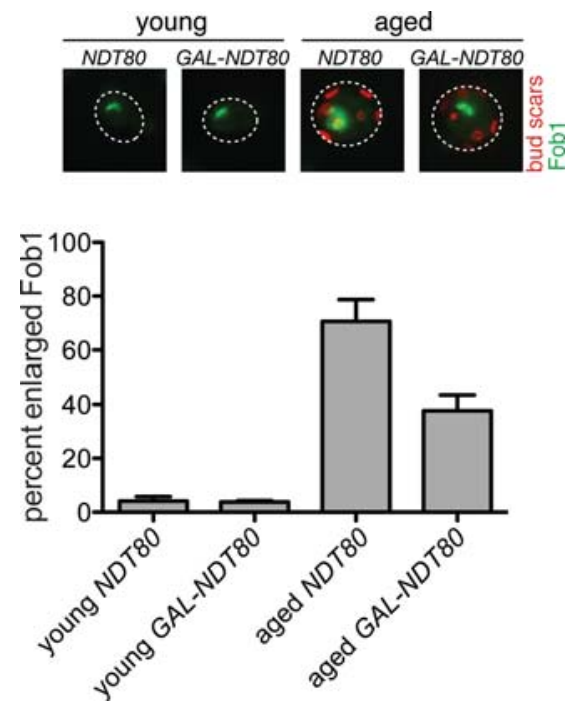

Figure 5. Nucleolar morphology is restored upon transient NDT80 induction. (Top) Representative images of Fob1-GFP (used as a marker for nucleolar/rDNA morphology) from A27507 (GAL4.ER, NDT80) and A27484 (GAL4.ER, GALNDT80) following $\beta$-estradiol treatment. Streptavidin-Alexa 568 (anti-biotin) shown in red and Fob1-GFP shown in green. (Bottom) The enlarged nucleolar morphology is scored $6 \mathrm{~h}$ after estradiol treatment. In each experiment, 100-200 cells were counted per sample. Error bars indicate standard deviation $(n=3)$. The average age for young GAL4-ER, NDT80 cells was $1.5 \pm 0.7$ generations; for aged GAL4-ER, NDT80 cells it was $15.5 \pm 4$ generations; for young GAL4-ER, GAL-NDT80 it was $1.5 \pm 0.8$ generations; and for aged GAL4-ER, GALNDT80 cells was $15.7 \pm 3.6$ generations.

suggests that changes in the nucleolus other than ERC accumulation trigger aging. Indeed, loss of nuclear and/or nucleolar integrity has severe functional consequences, including illegitimate recombination, defects in transcription and translation, and perturbations in nuclear-cytoplasmic trafficking (Guarente 1997; Kobayashi 2008; D'Angelo et al. 2009). In addition, loss of nucleolar integrity is a shared feature of premature aging syndromes such as Hutchinson-Gilford and Werner syndromes (Salk et al. 1985; De SandreGiovannoli et al. 2003). Finally, inhibition of TOR (target of rapamycin) signaling elicits a conserved starvation response to promote longevity. It is noteworthy that TOR inhibition also triggers rapid changes in nucleolar morphology (Tsang et al. 2007). Together, these studies point toward changes in nucleolar physiology as a critical source of aging. Understanding the impact of aging on the nucleolus, as well as the impact of gametogenesis on nucleolar structure and physiology, is thus critical.

Which aspects of nucleolar physiology could be affected by aging? The central function of the nucleolus is to make ribosomes; transcription of ribosomal DNA (rDNA) and assembly of the 40S and 60S ribosomal subunits take place in the nucleolus (Planta 1997). In budding yeast, $\sim 150$ rDNA gene copies are organized in the nucleolus as a single tandem array. Each rDNA repeat is $9.1 \mathrm{~kb}$ and contains the polymerase $\mathrm{I}$-transcribed 
35S pre-rRNA gene and the polymerase III-transcribed 5S rRNA gene (Planta 1997). In logarithmically growing budding yeast cells, $\sim 50 \%$ of the rDNA repeats are transcribed (Dammann et al. 1993). It is thought that the nontranscribed rDNA copies are required for maintaining the integrity of the rDNA locus by promoting the association of condensin complexes with the rDNA (Ide et al. 2010). It would be interesting to investigate whether rDNA transcription, silencing and/or rDNA condensation are affected during aging. Aged cells also exhibit increased rDNA instability (Sinclair and Guarente 1997; Lindstrom et al. 2011), suggestive of increased double-strand break formation and/or illegitimate recombination at the rDNA locus. Therefore, a detailed analysis of double-strand break formation and recombination at the rDNA in aged cells, as well as the impact of transient NDT80 induction on these processes, will be important.

Nucleolar architecture must also be investigated. The rDNA is linked to the nuclear periphery through associations of the rDNA-associated protein Lrs4 with two inner nuclear membrane proteins, Heh1 (also known as Src1) and Nur1, which are the yeast homologs of the mammalian LEM domain proteins (LBR, Emerin, and MAN1; Mekhail et al. 2008). In budding yeast, deletion of the genes encoding these proteins decreases life span, disrupts the nucleolus-nucleoplasm boundary, reduces perinuclear rDNA positioning, induces the formation of recombination foci, and destabilizes the rDNA repeats (Mekhail et al. 2008; Chan et al. 2011). These phenotypes are reminiscent of a $\operatorname{sir} 2 \Delta$ mutant with one significant exception: rDNA silencing is defective in the absence of Sir2, but not in heh1s or nurls mutants. First, this suggests that rDNA silencing cannot be the sole determinant in controlling recombination at the rDNA locus, and that correct perinuclear positioning and the architecture of the rDNA array are crucial for maintaining rDNA stability. Second, it suggests that changes in nucleolar architecture could be a source of aging. Interestingly, both Heh1 and Nur1 are induced during sporulation, and are likely Ndt80 targets (Chu et al. 1998; Primig et al. 2000).

The nucleolus has functions in addition to ribosome subunit biogenesis (Pederson and Tsai 2009; Warner and McIntosh 2009). This is highlighted by the fact that only $30 \%$ of the 4500 nucleolus-associated proteins identified through proteomic approaches have a function clearly related to the production of ribosome subunits (Ahmad et al. 2009). The other nucleolar functions include biogenesis of multiple RNPs, apoptosis, and viral infection. The nucleolus is also a storage unit for a number of cell cycle regulators including the protein phosphatase Cdc14 and the p53 regulator p19Arf (Visintin and Amon 2000). Disruption in the regulation of these processes could be responsible for the slowing of the cell cycle that is observed in aging yeast cells. In summary, it will be important to determine which of the numerous nucleolar functions are perturbed during aging, and whether and how the nucleolus is partitioned during mitosis, such that the mother cell retains the senescence factors but the daughter cell does not. Finally, how the nucleolus is restructured during sporulation and after transient NDT80 induction in vegetative cells will aid in determining how age-induced nucleolar changes contribute to aging.

The nucleolus may, however, not be the only target of gametogenesis-induced rejuvenation. Nuclear architecture, histone modifications, telomere structure, and mitochondrial biogenesis/degradation have also been shown to be affected by aging. For example, it is thought that, in budding yeast, the preexisting nuclear pores reside in the mother cells through the action of a septindependent lateral diffusion barrier (Shcheprova et al. 2008). In aged cells, nuclear pores deteriorate and become leaky (D'Angelo et al. 2009), which results in nuclear-cytoplasmic trafficking defects. Intriguingly, perturbations in mRNA export result in rapid fragmentation of nucleolar constituents, a phenotype that is also observed in aged cells (Thomsen et al. 2008). In addition, numerous subunits of the nuclear pore complex and associated factors are expressed during sporulation after NDT80 expression (G. Brar, pers. comm.), raising the possibility that nuclear pore remodeling could be part of gametogenesis-induced rejuvenation.

Changes in the posttranslational modifications of histones such as H4K16 acetylation have also been implicated in aging in budding yeast and have been linked to Sir2dependent longevity (Dang et al. 2009). Analysis of histone modifications during sporulation revealed specific changes in histone modifications (Krishnamoorthy et al. 2006; Govin et al. 2010a,b). In addition, the H3K4 demethylase Lsd-1 contributes to germline immortality in Caenorhabditis elegans (Katz et al. 2009). However, despite being up-regulated upon entry into sporulation (Chu et al. 1998; Primig et al. 2000), we found that the yeast homolog of $l s d-1, J H D 2$, is dispensable for gametogenesis-induced rejuvenation in budding yeast (Fig. 6). Thus, either histone demethylation is not important for life span resetting or redundant pathways exist in budding yeast.

Aging is also associated with mitochondrial dysfunction. Respiration affects some key determinants of

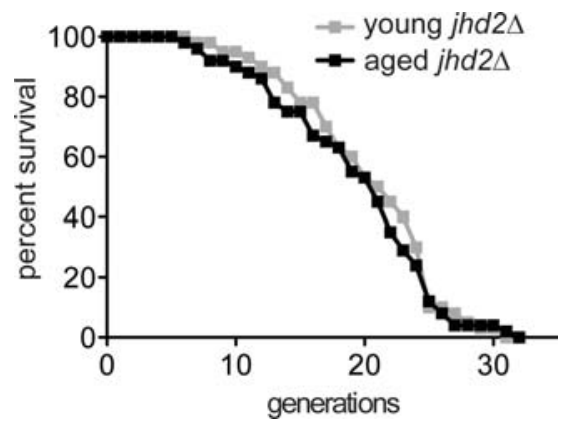

Figure 6. The histone H3K4 demethylase Jhd2 is dispensable for gametogenesis-induced rejuvenation. The postsporulation life span of young $(1.3 \pm 0.6$ generations $)$ and aged $(10 \pm 2.6$ generations) cells from a strain carrying a deletion of $\overline{J H D} 2$ (A24155) was compared by pedigree analysis. The experiment was performed using 15 tetrads ( $n=60$ spores) from each sample. Further technical details are available in Unal et al. (2011). 
longevity such as $\mathrm{NAD}^{+}$levels, amino acid metabolism, and reactive oxygen species. Interestingly, all of these metabolites are affected when cells, or organisms, are subjected to calorie restriction (Guarente 2008). In addition, mitochondrial dysfunction causes nuclear genome instability, probably by decreasing the production of iron sulfur cluster-containing proteins, which are required for maintenance of nuclear genome integrity (Veatch et al. 2009). Dysfunctional mitochondria are thought to be removed by a process called mitophagy, a mitochondrium-specific form of autophagy (Wang and Klionsky 2011). Interestingly, the crucial mitophagy factor Atg32 is up-regulated during sporulation (Chu et al. 1998; Primig et al. 2000), suggesting that mitochondrial remodeling occurs during gametogenesis.

Sporulation causes a fundamental restructuring of the cell. It brings about a dramatic change in gene expression and triggers large-scale protein degradation (Teichert et al. 1987, 1989; Chu et al. 1998; Primig et al. 2000). We speculate that this causes the purging of defective structures and organelles and brings about resetting of life span.

\section{DOES GAMETOGENESIS-INDUCED REJUVENATION OCCUR IN OTHER ORGANISMS?}

Work in Caenorhabditis elegans also links gametogenesis to longevity. In $C$. elegans, a number of longevity mutants in the insulin-like signaling pathway induce transcription of the germline specification factor pie- 1 in somatic cells. This soma to germline transformation is necessary for enhanced survival mediated by downregulation of insulin-like signaling and suggests that germline specific factors antagonize aging (Curran et al. 2009). Moreover, oxidatively damaged proteins, linked to aging diseases like Alzheimer's disease and cancer, are eliminated during gametogenesis in C. elegans (Goudeau and Aguilaniu 2011), similar to our observations in budding yeast (Fig. 2). These studies are consistent with gametogenesis-induced rejuvenation occurring in C. elegans.
Is there evidence that $\mathrm{Ndt} 80$ homologs could be involved in a potential rejuvenation mechanism in C. elegans? Ndt80 is most closely related to the p53 family of transcription factors. These transcription factors are critical for cells to mount a response to toxic conditions (i.e., DNA damage) that includes cell cycle arrest and apoptosis (Lane and Levine 2010). At the primary sequence level, Ndt80 is more similar to the p53 family member p63 than p53, specifically the TAp63 $\alpha$ isoform (Fig. 7). TAp63 $\alpha$ is enriched in the germline in mice and plays a role in oocyte quality control (Suh et al. 2006). Importantly, the C. elegans p53 homolog, cep-1, not only mediates the cellular response to DNA damage, but also regulates progression through gametogenesis (Derry et al. 2001). CEP-1 and Ndt80 share 17\% identity and $30 \%$ similarity at the amino acid sequence level (Fig. 7). CEP-1, like NDT80, is also more related to TAp $63 \alpha$ than p53 at the primary sequence level $(15 \%$ identity, 26\% similarity; Fig. 7). CEP-1 is expressed at low levels in somatic tissues and at higher levels in the germline and has been implicated in multiple stress responses in the soma, as well as apoptosis and meiotic chromosome segregation in the germline (Derry et al. 2001). The impact of cep-1 on longevity seems to be context-dependent; in conditions of high genotoxic stress, cep-1 reduces life span, presumably by inducing apoptosis in response to severe genotoxic stress. However, in nonstressed animals or under conditions that induce mild heat shock or oxidative stress, cep-1 promotes longevity (Ventura et al. 2009). The effect of cep-1 on longevity has so far been investigated only in the context of loss-of-function cep-1 mutants. A heat-shock-inducible cep-1 allele is available; however, this level of cep- 1 expression causes developmental arrest (Derry et al. 2001). Similarly, high levels of Ndt80 in budding yeast perturb growth and cell cycle progression, whereas lower levels of Ndt80 overexpression extends life span (Unal et al. 2011). It would be interesting to determine the consequences of low-level expression of cep-1 in the soma on C. elegans life span.

Perhaps the ancient function of the p53 family of transcription factors is to respond to double-strand break

\begin{tabular}{|c|c|c|c|c|c|c|c|c|}
\cline { 2 - 9 } \multicolumn{1}{c|}{} & \multicolumn{2}{c|}{ Ndt80 } & \multicolumn{2}{c|}{ Cep-1 } & \multicolumn{2}{c|}{ p53 } & \multicolumn{2}{c|}{ TA-p63 $\alpha$} \\
\hline Ndt80 & 100 & 100 & 17.4 & 30.5 & 3.5 & 5.4 & 14.3 & 24.1 \\
\hline Cep-1 & 17.4 & 30.5 & 100 & 100 & 11.5 & 19 & 15.2 & 25.6 \\
\hline p53 & 3.5 & 5.4 & 11.5 & 19 & 100 & 100 & 23.1 & 32.5 \\
\hline TA-p63 $\alpha$ & 14.3 & 24.1 & 15.2 & 25.6 & 23.1 & 32.5 & 100 & 100 \\
\hline
\end{tabular}

Figure 7. Sequence conservation between Ndt80, CEP-1, mouse p53, and TA-p63 $\alpha$. The Needleman-Wunsch pairwise alignment algorithm was used to determine the percentage identity (white boxes) and similarity (gray boxes) between Ndt80 (budding yeast), CEP-1 (nematode), p53 (mouse), and TA-p63 $\alpha$ (mouse). 
formation during meiosis and to mediate elimination of aging factors. This germline protective function of the p53 family, we speculate, was then adapted during evolution to accommodate general stress response and tumor suppressive functions.

Whether life span resetting occurs during gametogenesis in mammals is unclear. In mammals, a decrease in telomere length is associated with aging (Blackburn et al. 2006). Furthermore, during embryogenesis, telomerase is strongly activated at the morula/blastocyst transition. At this transition, telomeres are significantly elongated in murine and bovine embryos (Schaetzlein et al. 2004; Schaetzlein and Rudolph 2005), suggesting that telomere length resetting and thus life span resetting occur during early embryogenesis rather than during gametogenesis. A telomere resetting mechanism per se requires efficient net synthesis of telomeric DNA, which, in turn depends on high telomerase activity and high levels of shelterin, a complex involved in telomere protection. The genes encoding telomerase and the shelterin complex exhibit expression profiles typical of maternal genes, supporting the notion that the program to increase telomere lengths is present already in the egg (VizlinHodzic et al. 2009), and thus is regulated during gametogenesis. The impact of telomerase on life span is revealed by overexpression of telomerase in a cancer-resistant mouse model and by reintroduction of telomerase into a telomerase-deficient mouse, which causes a rescue of age-related phenotypes (Tomas-Loba et al. 2008; Jaskelioff et al. 2010). Together, these results highlight the importance of telomere homeostasis for longevity in mammals. However, whether other age-induced cellular changes such as nuclear/nucleolar perturbations, protein aggregation, and mitochondrial aberrations are eliminated during gametogenesis or embryogenesis remains to be determined.

\section{CONCLUSIONS}

In budding yeast, gamete formation resets replicative life span and eliminates age-induced cellular damage. Given that resetting of life span occurs in all eukaryotes with each generation, it will be interesting to investigate whether our findings extend to other species. Our characterization of age-associated events during gametogenesis and of the consequences of ectopic Ndt80 expression on aging-associated markers furthermore creates a unique opportunity to dissect the molecular causes of aging. For example, elimination of Hsp104-eGFP aggregates and ERCs does not appear to be required for extension of life span brought about by NDT80 induction, but changes in nucleolar function and/or structure may be important. Finally, our studies raise the interesting possibility that a transient induction of the gametogenesis program in somatic cells increases life span. Understanding the mechanisms that mediate gametogenesis-induced rejuvenation will not only provide insights into how gametogenesis ensures cellular fitness but could also facilitate the development of new reprogramming and regeneration strategies.

\section{ACKNOWLEDGMENTS}

We thank Michelle Attner and Matthew Miller for comments on the manuscript. Research was supported by National Institutes of Health grant GM62207 to A.A. A.A. is also a Howard Hughes Medical Institute investigator. E.Ü. is a fellow of the Jane Coffin Childs Memorial Fund.

\section{REFERENCES}

Ahmad Y, Boisvert FM, Gregor P, Cobley A, Lamond AI. 2009. NOPdb: Nucleolar Proteome Database-2008 update. Nucleic Acids Res 37: 181-184.

Blackburn EH, Greider CW, Szostak JW. 2006. Telomeres and telomerase: The path from maize, Tetrahymena and yeast to human cancer and aging. Nat Med 12: 1133-1138.

Chan JN, Poon BP, Salvi J, Olsen JB, Emili A, Mekhail K. 2011. Perinuclear cohibin complexes maintain replicative life span via roles at distinct silent chromatin domains. Devl Cell 20: $867-879$

Chu S, DeRisi J, Eisen M, Mulholland J, Botstein D, Brown PO, Herskowitz I. 1998. The transcriptional program of sporulation in budding yeast. Science 282: 699-705.

Curran SP, Wu X, Riedel CG, Ruvkun G. 2009. A soma-to-germline transformation in long-lived Caenorhabditis elegans mutants. Nature 459: 1079-1084.

Dammann R, Lucchini R, Koller T, Sogo JM. 1993. Chromatin structures and transcription of rDNA in yeast Saccharomyces cerevisiae. Nucleic Acids Res 21: 2331-2338.

Dang W, Steffen KK, Perry R, Dorsey JA, Johnson FB, Shilatifard A, Kaeberlein M, Kennedy BK, Berger SL. 2009. Histone $\mathrm{H} 4$ lysine 16 acetylation regulates cellular lifespan. Nature 459: 802-807.

D’Angelo MA, Raices M, Panowski SH, Hetzer MW. 2009. Age-dependent deterioration of nuclear pore complexes causes a loss of nuclear integrity in postmitotic cells. Cell 136: $284-295$.

Derry WB, Putzke AP, Rothman JH. 2001. Caenorhabditis elegans p53: Role in apoptosis, meiosis, and stress resistance. Science 294: 591-595.

De Sandre-Giovannoli A, Bernard R, Cau P, Navarro C, Amiel J, Boccaccio I, Lyonnet S, Stewart CL, Munnich A, Le Merrer M, Levy N. 2003. Lamin a truncation in Hutchinson-Gilford progeria. Science 300: 2055.

Ephrussi A, Lehmann R. 1992. Induction of germ cell formation by oskar. Nature 358: 387-392.

Erjavec N, Larsson L, Grantham J, Nystrom T. 2007. Accelerated aging and failure to segregate damaged proteins in Sir2 mutants can be suppressed by overproducing the protein aggregation-remodeling factor Hsp104p. Genes Devl 21: 2410-2421.

Fabrizio P, Longo VD. 2003. The chronological life span of Saccharomyces cerevisiae. Aging Cell 2: 73-81.

Fuchs J, Loidl J. 2004. Behaviour of nucleolus organizing regions (NORs) and nucleoli during mitotic and meiotic divisions in budding yeast. Chromosome Res 12: 427-438.

Goudeau J, Aguilaniu H. 2011. Carbonylated proteins are eliminated during reproduction in C. elegans. Aging Cell 9: $991-1003$.

Govin J, Dorsey J, Gaucher J, Rousseaux S, Khochbin S, Berger SL. 2010a. Systematic screen reveals new functional dynamics of histones $\mathrm{H} 3$ and $\mathrm{H} 4$ during gametogenesis. Genes Dev 24: $1772-1786$.

Govin J, Schug J, Krishnamoorthy T, Dorsey J, Khochbin S, Berger SL. 2010b. Genome-wide mapping of histone H4 serine-1 phosphorylation during sporulation in Saccharomyces cerevisiae. Nucleic Acids Res 38: 4599-4606.

Guarente L. 1997. Link between aging and the nucleolus. Genes Devl 11: 2449-2455. 
Guarente L. 2008. Mitochondria - A nexus for aging, calorie restriction, and sirtuins? Cell 132: 171-176.

Ide S, Miyazaki T, Maki H, Kobayashi T. 2010. Abundance of ribosomal RNA gene copies maintains genome integrity. Science 327: 693-696.

Jaskelioff M, Muller FL, Paik JH, Thomas E, Jiang S, Adams AC, Sahin E, Kost-Alimova M, Protopopov A, Cadinanos J, et al. 2010. Telomerase reactivation reverses tissue degeneration in aged telomerase-deficient mice. Nature 469: $102-106$.

Johnson FB, Sinclair DA, Guarente L. 1999. Molecular biology of aging. Cell 96: 291-302.

Katz DJ, Edwards TM, Reinke V, Kelly WG. 2009. A C. elegans LSD1 demethylase contributes to germline immortality by reprogramming epigenetic memory. Cell 137: 308-320.

Kirkwood TB. 1987. Immortality of the germ-line versus disposability of the soma. Basic Life Sci 42: 209-218.

Kobayashi T. 2008. A new role of the rDNA and nucleolus in the nucleus - rDNA instability maintains genome integrity. Bioessays 30: $267-272$.

Krishnamoorthy T, Chen X, Govin J, Cheung WL, Dorsey J, Schindler K, Winter E, Allis CD, Guacci V, Khochbin S, Fuller MT, et al. 2006. Phosphorylation of histone H4 Ser1 regulates sporulation in yeast and is conserved in fly and mouse spermatogenesis. Genes Devl 20: 2580-2592.

Lane D, Levine A. 2010. p53 Research: The past thirty years and the next thirty years. Cold Spring Harb Perspect Biol 2: a000893.

Lindstrom DL, Leverich CK, Henderson KA, Gottschling DE. 2011. Replicative age induces mitotic recombination in the ribosomal RNA gene cluster of Saccharomyces cerevisiae. PLoS Genet 7: e1002015.

Liu B, Larsson L, Caballero A, Hao X, Oling D, Grantham J, Nystrom T. 2010. The polarisome is required for segregation and retrograde transport of protein aggregates. Cell 140: 257-267.

Marston AL, Amon A. 2004. Meiosis: cell-cycle controls shuffle and deal. Nat Rev Mol Cell Biol 5: 983-997.

Mekhail K, Seebacher J, Gygi SP, Moazed D. 2008. Role for perinuclear chromosome tethering in maintenance of genome stability. Nature 456: 667-670.

Morimoto RI. 2008. Proteotoxic stress and inducible chaperone networks in neurodegenerative disease and aging. Genes Dev 22: $1427-1438$.

Mortimer RK, Johnston JR. 1959. Life span of individual yeast cells. Nature 183: 1751-1752.

Neiman AM. 2005. Ascospore formation in the yeast Saccharomyces cerevisiae. Microbiol Mol Biol Rev 69: $565-584$.

Nystrom T. 2005. Role of oxidative carbonylation in protein quality control and senescence. EMBO J 24: 1311-1317.

Park HO, Bi E. 2007. Central roles of small GTPases in the development of cell polarity in yeast and beyond. Microbiol Mol Biol Rev 71: 48-96.

Pederson T, Tsai RY. 2009. In search of nonribosomal nucleolar protein function and regulation. $J$ Cell Biol 184: 771-776.

Planta RJ. 1997. Regulation of ribosome synthesis in yeast. Yeast 13: $1505-1518$.

Primig M, Williams RM, Winzeler EA, Tevzadze GG, Conway AR, Hwang SY, Davis RW, Esposito RE. 2000. The core meiotic transcriptome in budding yeasts. Nat Genet 26: $415-423$.

Salk D, Au K, Hoehn H, Martin GM. 1985. Cytogenetic aspects of Werner syndrome. Adv Exp Med Biol 190: $541-546$.
Schaetzlein S, Rudolph KL. 2005. Telomere length regulation during cloning, embryogenesis and ageing. Reprod Fertil Dev 17: 85-96.

Schaetzlein S, Lucas-Hahn A, Lemme E, Kues WA, Dorsch M, Manns MP, Niemann H, Rudolph KL. 2004. Telomere length is reset during early mammalian embryogenesis. Proc Natl Acad Sci 101: 8034-8038.

Shcheprova Z, Baldi S, Frei SB, Gonnet G, Barral Y. 2008. A mechanism for asymmetric segregation of age during yeast budding. Nature 454: 728-734

Sinclair DA, Guarente L. 1997. Extrachromosomal rDNA circles - A cause of aging in yeast. Cell 91: 1033-1042.

Sinclair DA, Mills K, Guarente L. 1997. Accelerated aging and nucleolar fragmentation in yeast sgs1 mutants. Science 277: $1313-1316$.

Sinclair D, Mills K, Guarente L. 1998. Aging in Saccharomyces cerevisiae. Annu Rev Microbiol 52: 533-560.

Smeal T, Claus J, Kennedy B, Cole F, Guarente L. 1996. Loss of transcriptional silencing causes sterility in old mother cells of S. cerevisiae. Cell 84: 633-642.

Suh EK, Yang A, Kettenbach A, Bamberger C, Michaelis AH, Zhu Z, Elvin JA, Bronson RT, Crum CP, McKeon F. 2006. p63 protects the female germ line during meiotic arrest. Nature 444: 624-628.

Teichert U, Mechler B, Muller H, Wolf DH. 1987. Protein degradation in yeast. Biochem Soc Trans 15: 811-815.

Teichert U, Mechler B, Muller H, Wolf DH. 1989. Lysosomal (vacuolar) proteinases of yeast are essential catalysts for protein degradation, differentiation, and cell survival. $J$ Biol Chem 264: 16037-16045.

Tessarz P, Schwarz M, Mogk A, Bukau B. 2009. The yeast $\mathrm{AAA}+$ chaperone Hsp104 is part of a network that links the actin cytoskeleton with the inheritance of damaged proteins. Mol Cell Biol 29: 3738-3745.

Thomsen R, Saguez C, Nasser T, Jensen TH. 2008. General, rapid, and transcription-dependent fragmentation of nucleolar antigens in S. cerevisiae mRNA export mutants. RNA 14: $706-716$

Tomas-Loba A, Flores I, Fernandez-Marcos PJ, Cayuela ML, Maraver A, Tejera A, Borras C, Matheu A, Klatt P, Flores JM, et al. 2008. Telomerase reverse transcriptase delays aging in cancer-resistant mice. Cell 135: 609-622.

Tsang CK, Li H, Zheng XS. 2007. Nutrient starvation promotes condensin loading to maintain rDNA stability. EMBO J 26: $448-458$.

Unal E, Kinde B, Amon A. 2011. Gametogenesis eliminates age-induced cellular damage and resets life span in yeast. Science 332: 1554-1557.

Veatch JR, McMurray MA, Nelson ZW, Gottschling DE. 2009. Mitochondrial dysfunction leads to nuclear genome instability via an iron-sulfur cluster defect. Cell 137: 1247-1258.

Ventura N, Rea SL, Schiavi A, Torgovnick A, Testi R, Johnson TE. 2009. p53/CEP-1 increases or decreases lifespan, depending on level of mitochondrial bioenergetic stress. Aging Cell 8: 380-393.

Visintin R, Amon A. 2000. The nucleolus: the magician's hat for cell cycle tricks. Curr Opin Cell Biol 12: 372-377.

Vizlin-Hodzic D, Ryme J, Simonsson S, Simonsson T. 2009. Developmental studies of Xenopus shelterin complexes: the message to reset telomere length is already present in the egg. FASEB J 23: 2587-2594.

Wang K, Klionsky DJ. 2011. Mitochondria removal by autophagy. Autophagy 3: 297-300.

Warner JR, McIntosh KB. 2009. How common are extraribosomal functions of ribosomal proteins? Mol Cell 34: 3-11. 


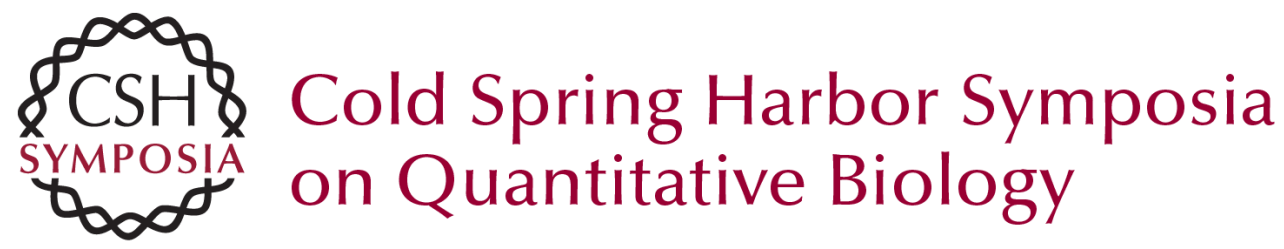

\section{Gamete Formation Resets the Aging Clock in Yeast}

E. Ünal and A. Amon

Cold Spring Harb Symp Quant Biol 2011 76: 73-80 originally published online September 2, 2011

Access the most recent version at doi:10.1101/sqb.2011.76.011379

References This article cites 61 articles, 21 of which can be accessed free at: http://symposium.cshlp.org/content/76/73.full.html\#ref-list-1

\section{License}

Email Alerting

Receive free email alerts when new articles cite this article - sign up in

Service the box at the top right corner of the article or click here. 